\title{
In situ quantitative diagnosis of insulated building walls using passive infrared thermography
}

by M. H.A. Larbi Youcef*, V. Feuillet*, L. Ibos*, Y. Candau*, P. Balcon**, A. Filloux***

\author{
*Université Paris-Est, CERTES, 61 avenue du Général de Gaulle, 94010 Créteil, France \\ ${ }^{*}$ FLIR Advanced Thermal Systems, 19 boulevard Bidault, 77183 Croissy Beaubourg, France \\ ${ }^{* * *}$ Alpheeis, Espace Beethoven, 1200 route des lucioles, 06560 Valbonne, France
}

\section{Abstract}

This study concerns the application of passive infrared thermography to obtain a quantitative estimation of thermal insulation of building walls. In situ measurements were performed during several days. Thermographic measuremenats were completed by additional measurements, particularly of atmospheric parameters. The thickness or the thermal conductivity of the insulation layer is estimated by inverse method. The influence of the measurement duration or sampling frequency on the identified parameters and uncertainties is discussed.

\section{Introduction}

This work follows up the NADIIAH project which was conducted by the same consortium. This previous project led to the establishment of a protocol of measurement of the total thermal resistance of insulated interior wall by using active infrared thermography. The feasibility of this application in laboratory was proven for different commercial multilayered panels fixed onto a building wall [1].

The present study (DPE_IITI project) aims at applying passive infrared thermography for the in situ quantitative diagnosis of insulated building walls. This work is financially supported by the French agency for environment and energy saving (ADEME). In literature, only a few quantitative diagnoses by means of passive infrared thermography exist. Recently a complete methodology based on the infrared thermovision technique has been developed to reach quantitative data of thermal transmittances of building envelopes [2]. Another interesting approach consists in investigating both visual and IR images in the same study [3] to yield a diagnosis of various façades with a significant reduction of analysis time per evaluation.

In this work an experimental test bench was used in real conditions to examine a multi-layered wall located in a primary school in the town of Noisiel, near Paris. Experimental results have been combined with different numerical heat transfer models to evaluate the insulation level of the wall.

\section{Experimental setup}

Figure 1 shows exterior and interior views of the in situ experimental test bench. An acquisition module located inside the building is connected to several sensors to measure continuously a set of physical parameters:

- atmospheric parameters (temperature, pressure, relative humidity, vertical and horizontal solar heat flux) acquired thanks to an homemade weather station;

- indoor and outdoor wall temperatures measured by means of infrared cameras and thermocouples.
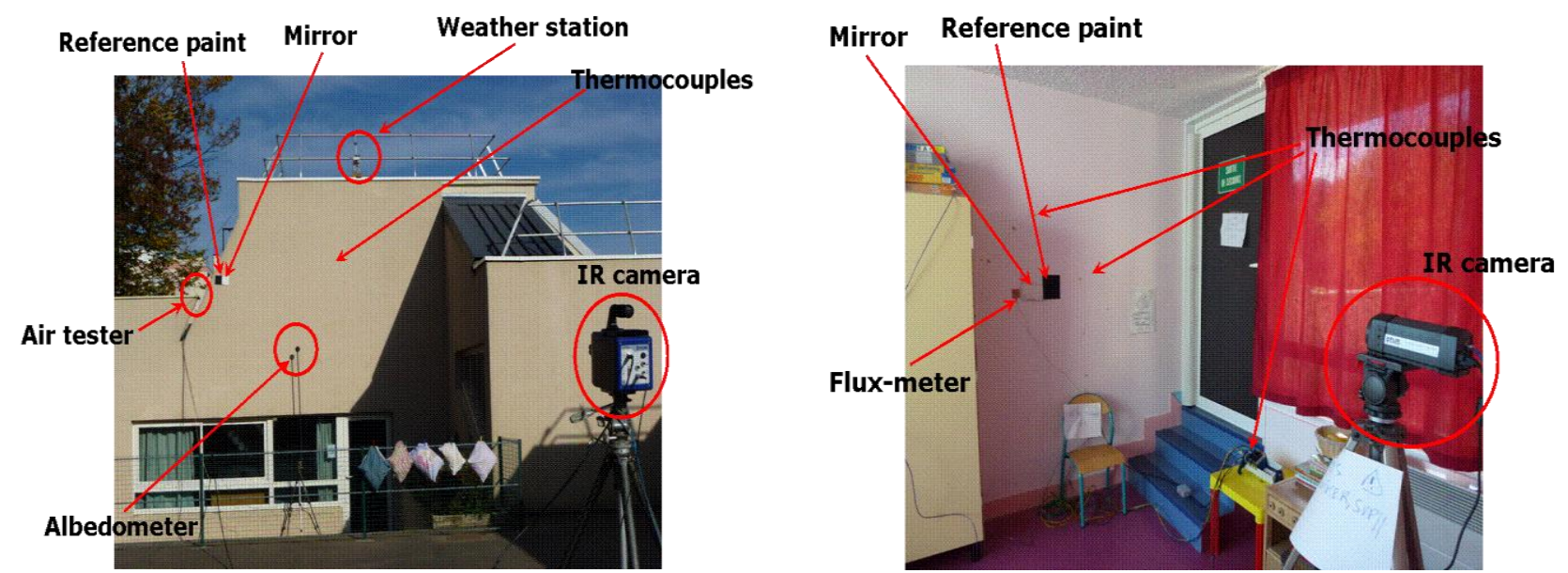

Fig. 1. Exterior and interior views of the in situ experimental test bench 


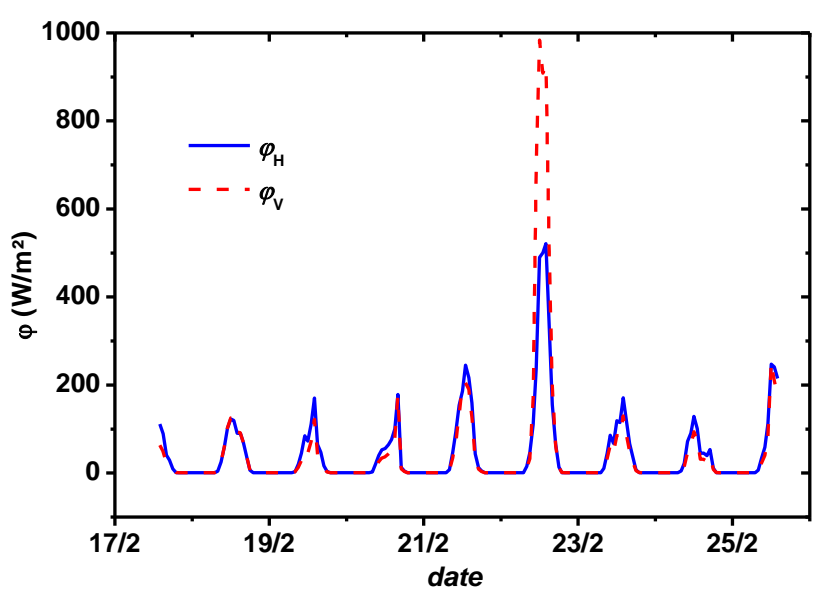

Fig. 2. Horizontal and vertical solar flux density measured during the campaign of February 2011
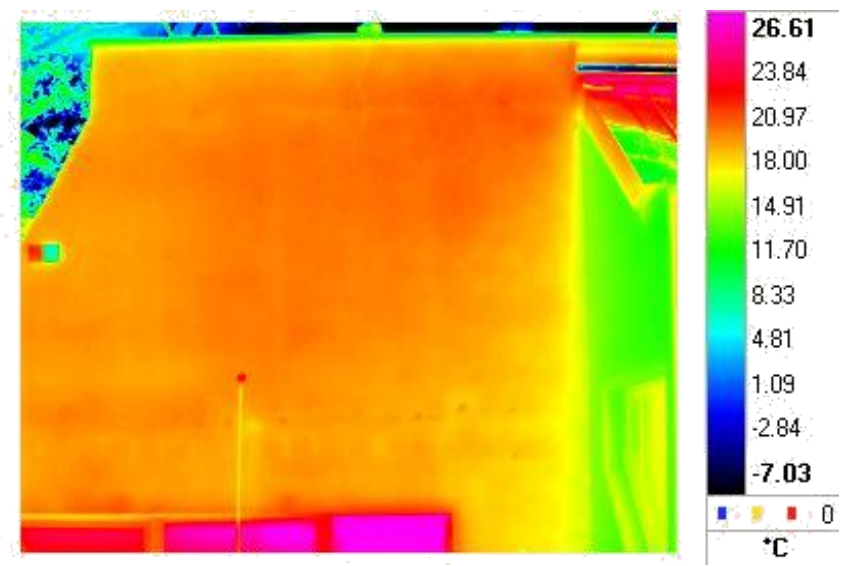

Fig. 4. Outdoor IR thermogram of the studied wall (FLIR SC7300 camera)

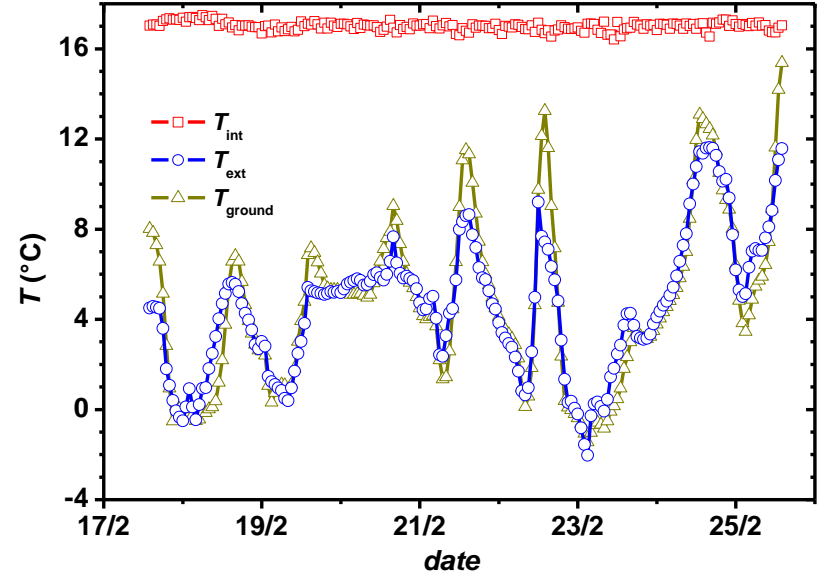

Fig. 3. Interior and exterior air temperature measured during the campaign of February 2011; comparison to exterior ground temperature

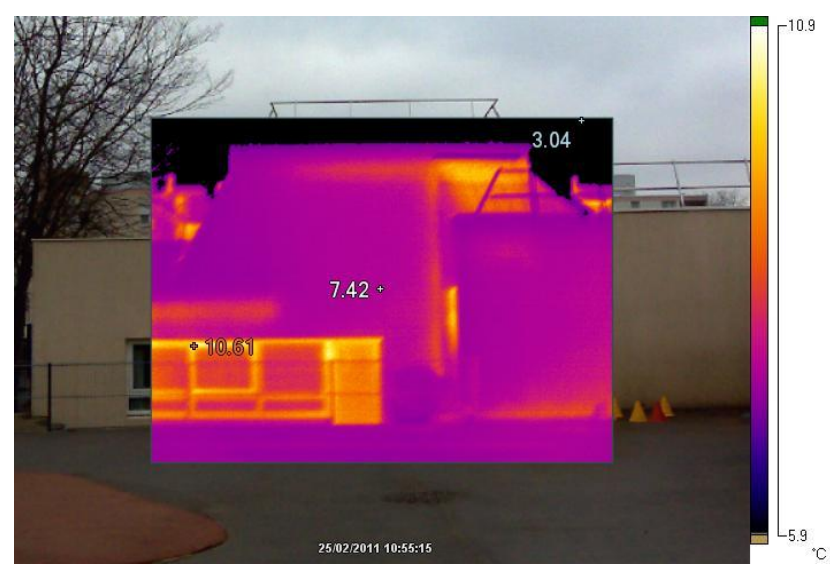

Fig. 5. Outdoor IR thermogram of the studied wall (Fluke TI32 camera with fusion mode)

Several punctual additional measurements are also performed such as exterior surface albedo and wall surface heat flux. The connexion between the acquisition module and the different sensors is realized either by wires (interior sensors) or Wifi transmission (exterior sensors). This experimental setup is automatically controlled by a Labview $^{\odot}$ application with a sampling period of 1 minute. The two measurement campaign durations are 8 days (February 2011) and 4 days (October 2011).

Measurements of solar heat flux density (horizontal and vertical values) for the measurement campaign of February 2011 are presented in figure 2. This campaign includes variable weather conditions, cloudy and rainy days and a sunny day (22/02). The evolution of interior and exterior air temperatures during the same period are presented in figure 3, along with the evolution of the outdoor ground temperature. During this campaign, air temperature inside the school remains close to $17^{\circ} \mathrm{C}$; this was a holiday period and the school was not used (winter holidays). The air temperature inside all rooms is regulated. The exterior air temperature shows strong variations, particularly during sunny days. The interior-exterior air temperature gradient is varying between 5 and $20^{\circ} \mathrm{C}$.

Indoor thermographic measurements were performed using an uncooled-microbolometer camera (FLIR A325) whereas outdoor thermographic measurements are performed using a LWIR cooled camera (FLIR SC 7300). Additional punctual thermal imaged are recorded using a portable camera (FLUKE TI32) designed for maintenance applications. Examples of IR image of the outdoor wall under study are presented in figures 4 and 5 . The evolution of the temperature of exterior and interior wall surfaces is presented in figure 6 . As observed for air temperature measurements, the interior wall surface temperature remains nearly constant to a value close to $15.5^{\circ} \mathrm{C}$ whereas strong variations of exterior wall surface temperature are noted. During the sunny day (22/02), we observe a negative value of the temperature gradient due to an important increase of the exterior wall surface temperature due to the high value of the solar flux density during this day. Thus, interior-exterior temperature gradient varies between -7 and $13^{\circ} \mathrm{C}$, during the whole campaign. 


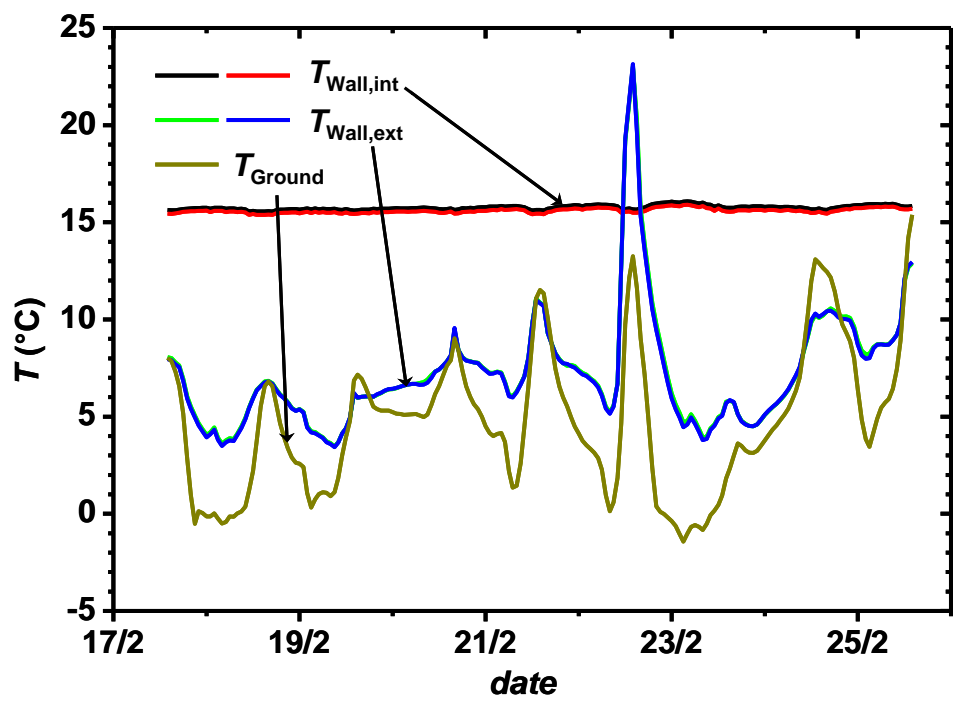

Fig. 6. Evolution of wall surface temperature (exterior and interior values); comparison to ground temperature

Table 1. Properties of the investigated wall [1]

\begin{tabular}{|l|l|l|l|l|}
\hline Layer $i$ & $\begin{array}{l}\text { Thickness } \\
e_{i}(\mathrm{~cm})\end{array}$ & $\begin{array}{l}\text { Thermal conductivity } \lambda_{i} \\
\left(\mathrm{~W} \cdot \mathrm{m}^{-1} \cdot \mathrm{K}^{-1}\right)\end{array}$ & $\begin{array}{l}\text { Density } \\
\rho_{i}\left(\mathrm{~kg} \cdot \mathrm{m}^{-3}\right)\end{array}$ & $\begin{array}{l}\text { Heat capacity } \\
c_{i}\left(\mathrm{~J}_{\mathrm{K}} \mathrm{K}^{-1} \cdot \mathrm{kg}^{-1}\right)\end{array}$ \\
\hline Concrete $(i=1)$ & 16 & 1.15 & 1900 & 1000 \\
\hline Expanded Polystyrene $(i=2)$ & 4 & 0.038 & 20 & 1450 \\
\hline Concrete $(i=3)$ & 16 & 1.15 & 1900 & 1000 \\
\hline
\end{tabular}

\section{Analysis method and results}

\subsection{Presentation of the identification procedure}

The previous measured parameters are associated with heat transfer numerical modeling (finite elements or finite differences) in order to estimate the insulation level of the wall. A schematic view of the wall structure and heat exchanges is presented in Figure 7. The investigated wall is constituted by two concrete layers separated by an insulating layer made of expanded polystyrene whose thickness is known $(4 \mathrm{~cm})$. Properties of materials considered are reported in table 1 . The analysis step aims at estimating the different modeling unknown parameters, especially the properties of the insulating layer. This identification is achieved by solving the corresponding inverse problem formulated in the least-squares sense. It consists in minimizing the functional that links the measured and estimated temperatures computed thanks to the heat transfer modeling by using Levenberg-Marquardt algorithm [4].

The unknown parameters estimated by inverse method are:

- the exterior and interior heat exchange coefficient values, respectively noted $h_{\text {ext }}$ and $h_{\text {int; }}$; these parameters are considered as constant during the whole measurement campaign: in fact we consider that using a mean value of these parameters is sufficient to obtain a good fitting of experimental data;

- the absorption factor of the wall surface $\alpha$;

- the insulating layer thickness $e_{2}$ (if its thermal conductivity is fixed) or its thermal conductivity $\lambda_{2}$ (if the thickness is fixed).

Initial values of identified parameters are: $h_{e x t}=10 \mathrm{~W} \cdot \mathrm{m}^{-2} \cdot \mathrm{K}^{-1} ; h_{\mathrm{int}}=5 \mathrm{~W} \cdot \mathrm{m}^{-2} \cdot \mathrm{K}^{-1} ; \alpha=0.3 ; e_{2}=6 \mathrm{~cm}$

The first results showed that it was possible to estimate either the thickness or the thermal conductivity of the insulating layer by analyzing the measurement data obtained for several consecutive days. For instance figure 8 shows the result of the estimation of the thermal conductivity of the insulating layer. Estimations converge to a value close to the thermal conductivity of expanded polystyrene (see table 1 ).

A preliminary study using data obtained from numerical simulations has shown that the greatest sensitivity to parameters to estimate was obtained by considering the temperature gradient between interior and exterior wall surface temperature, instead of considering only interior or exterior surface temperatures. This result was confirmed by estimations obtained considering experimental data of the campaign of February 2011. Table 2 presents a comparison of estimated parameter values obtained considering:

- the exterior wall surface temperature only;

- the interior wall surface temperature only;

- the gradient between both interior and exterior wall surface temperatures. 
These values were obtained by considering experimental data for the whole measurement campaign of February 2011.

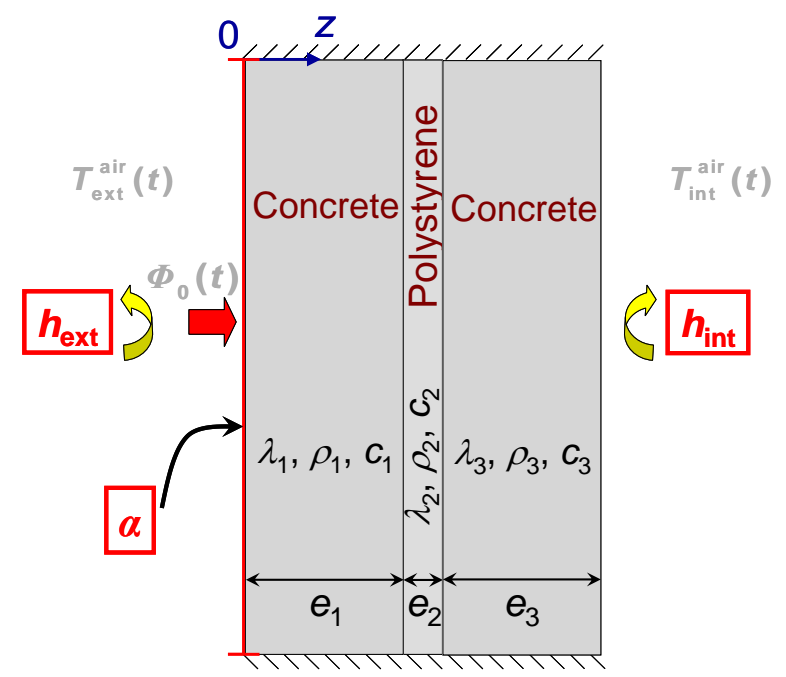

Fig. 7. Schematic representation of the wall structure and heat exchanges

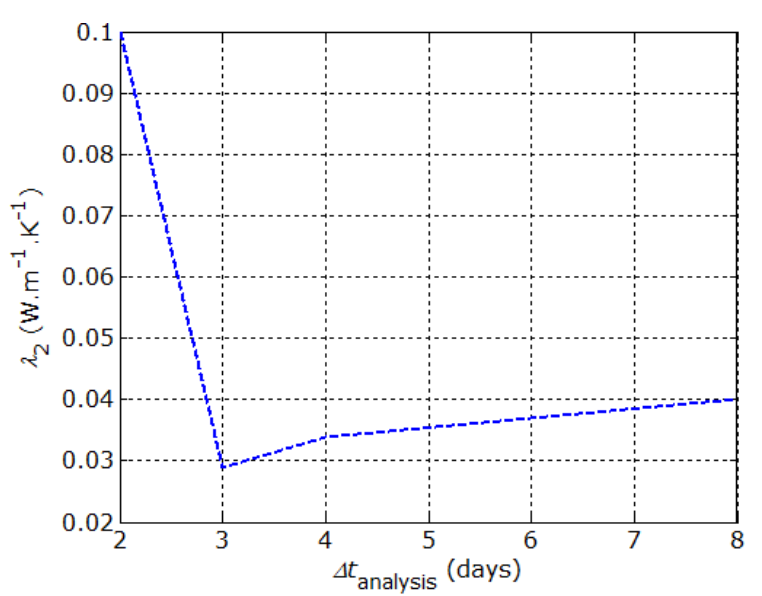

Fig. 8. Estimation of the insulating layer thickness as a function of the analysis duration

Table 2. Estimation results obtained by considering only exterior or interior wall surface temperatures or interior-exterior wall surface temperature gradient

\begin{tabular}{|c|c|c|c|c|}
\hline Considered data & $h_{\text {ext }}\left(\mathrm{W} \cdot \mathrm{m}^{-2} \cdot \mathrm{K}^{-1}\right)$ & $h_{\text {int }}\left(\mathrm{W} \cdot \mathrm{m}^{-2} \cdot \mathrm{K}^{-1}\right)$ & $\alpha$ & $e_{2}(\mathrm{~cm})$ \\
\hline$T_{\mathrm{ext}}$ & $9.06(0.05)$ & $11.33(0.47)$ & $0.53(0.02)$ & $2.23(0.05)$ \\
\hline$T_{\text {int }}$ & $7.94(0.03)$ & $6.33(0.02)$ & $0.32(0.01)$ & $5.47(0.03)$ \\
\hline$T_{\text {int }}-T_{\mathrm{ext}}$ & $9.25(0.05)$ & $4.72(0.09)$ & $0.27(0.01)$ & $4.40(0.08)$ \\
\hline
\end{tabular}

(a)

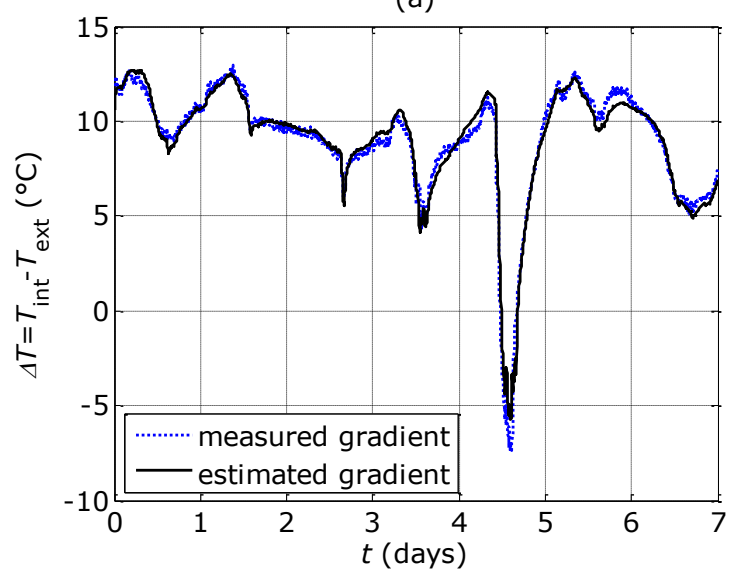

(b)

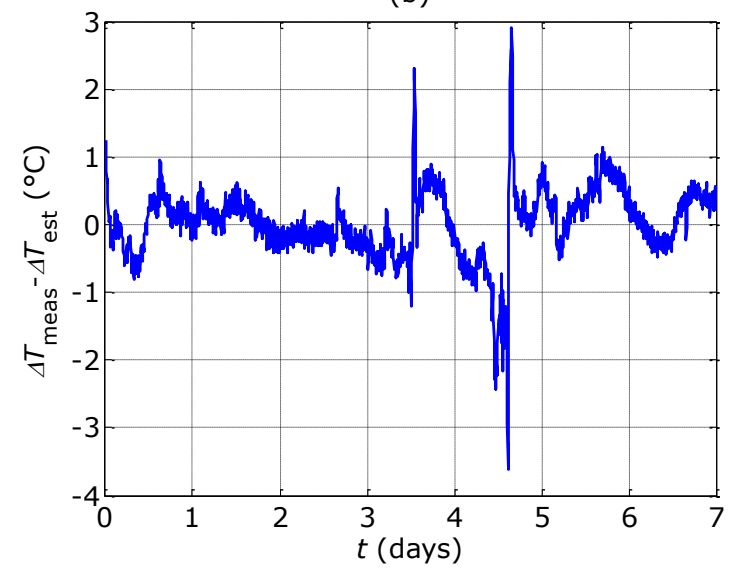

Figure 9. Identification results using the temperature gradient $\Delta T=T_{\text {int }}-T_{\text {ext }}$ during the whole measurement campaign of february 2011: (a) Comparison of measured and estimated temperature gradients, (b) Corresponding residuals

A comparison of estimated and measured temperature gradients is presented in figure 9 . We observe very small values of residuals (lower than $\pm 0.5^{\circ} \mathrm{C}$ ) except for the sunny day. Values of estimated parameters obtained using the temperature gradient are compatible with possible values of these parameters. The insulation layer thickness is close to the expected value. A posterior measurement of the wall surface albedo has provided a value of parameter a close to 0.45 . The discrepancy between this value and the estimated one can be explained by the position of solar flux sensors too far from the wall surface studied. This could also explain the high values of temperature gradient residuals observed in figure $9(\mathrm{~b})$ during the sunny day, i.e. when rapid variations of the solar flux density are observed. 


\subsection{Influence of analysis duration}

Influence of measurement duration on the estimation results was investigated by considering increasing analysis time end ( 1 day to 7 days). Estimation results are presented in figure 10 . We observe a small variation of the estimated values of the heat exchange coefficients. Difference between expected and estimated value of insulation layer thickness remains lower than $20 \%$ even when considering only one day of measurement. On the contrary, wall surface absorption coefficient shows important variations, particularly when the sunny day $\left(5^{\text {th }}\right.$ one) is considered. This also coincides with a sharp increase of the residual norm. Finally, statistical uncertainties lower than $5 \%$ are obtained using only the first day of measurement. These uncertainties falls around 2 and $3 \%$ for analyses considering 3 days of measurement or more, except for the wall surface absorption coefficient.

(a)

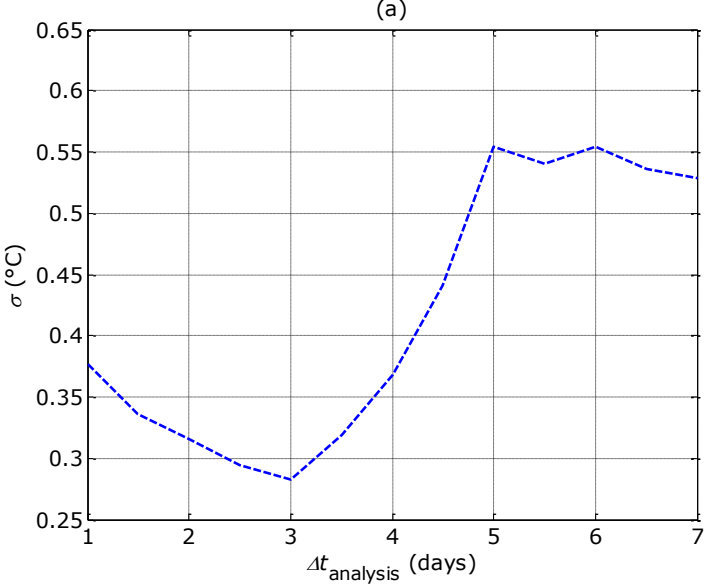

(c)

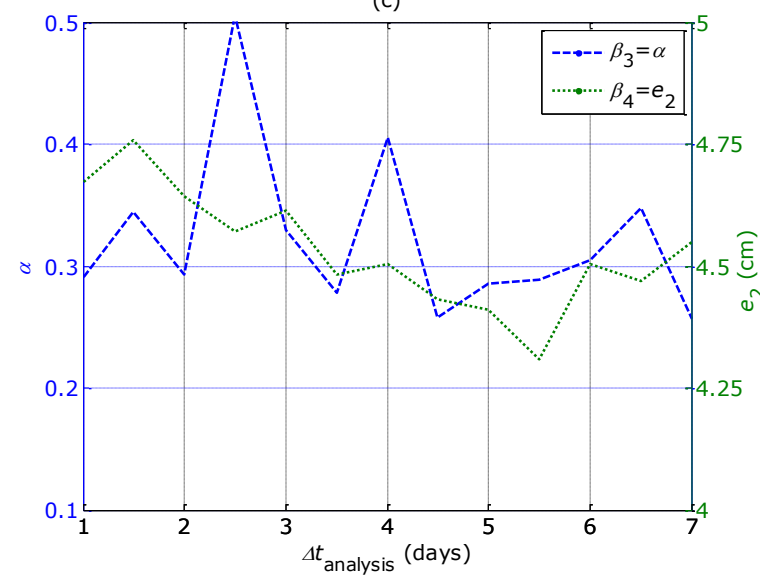

(b)

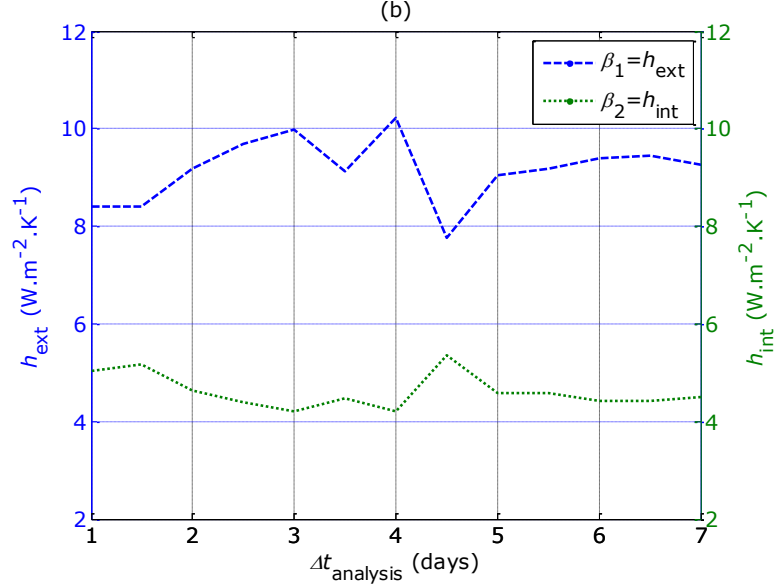

(d)

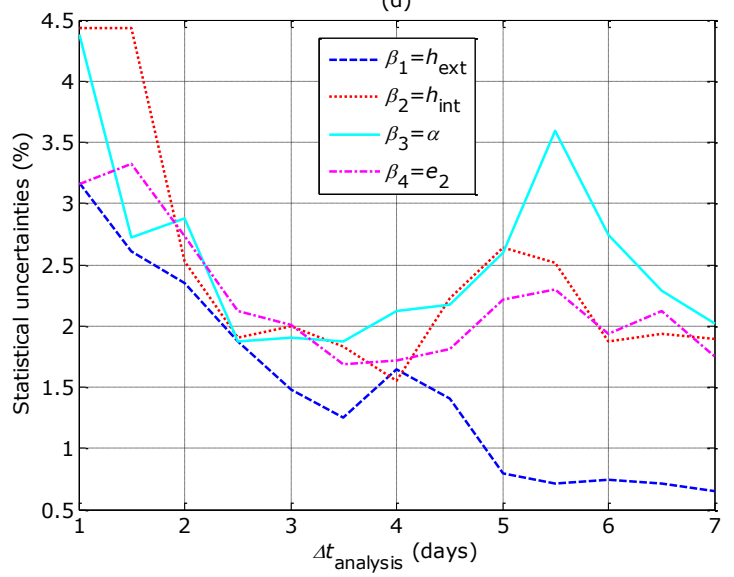

Figure 10. Identification results using the temperature gradient $\Delta T=T_{\text {int }}-T_{\text {ext }}$ as a function of duration analysis: (a) residual norm, (b) estimated parameters $h_{\text {ext }}$ and $h_{\text {int, }}(c)$ estimated parameters $\alpha$ et $e_{2}$, (d) statistical uncertainties on estimated parameters

\subsection{Influence of sampling frequency}

Influence of sampling frequency on the estimation results was investigated by considering increasing values of sampling period (from 5 to 300 minutes). In all cases, the measurement duration considered is seven days. Estimation results are presented in figure 11 . We observe only small variations of the estimated values of heat exchange coefficients and insulation layer thickness for sampling periods lower than or equal to four hours. A strong increase of residual norm and statistical uncertainties on these parameters is also observed. A good estimation of the wall surface absorption coefficient requires only short values of sampling period. However, variations of the estimations of this parameter have a marginal influence on the estimated values of the three other parameters. For future inspections this parameter will be fixed to a value measured using a home-made portable albedometer. 
(a)

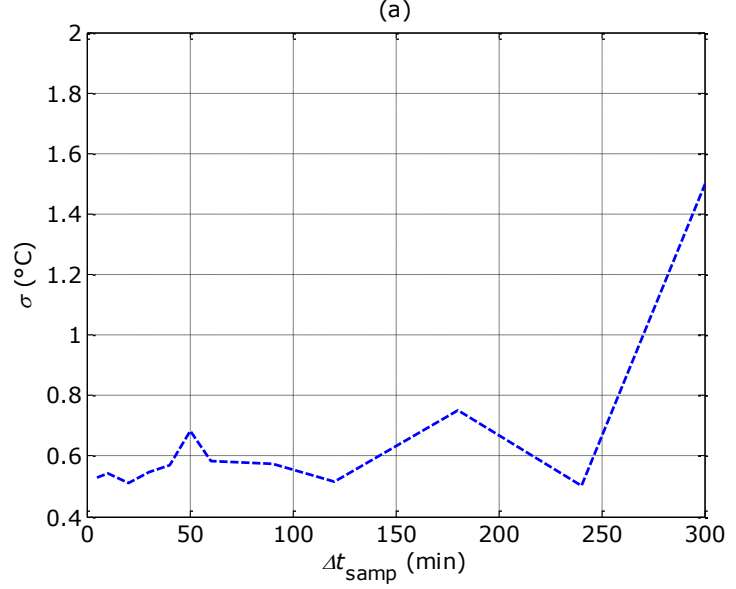

(c)

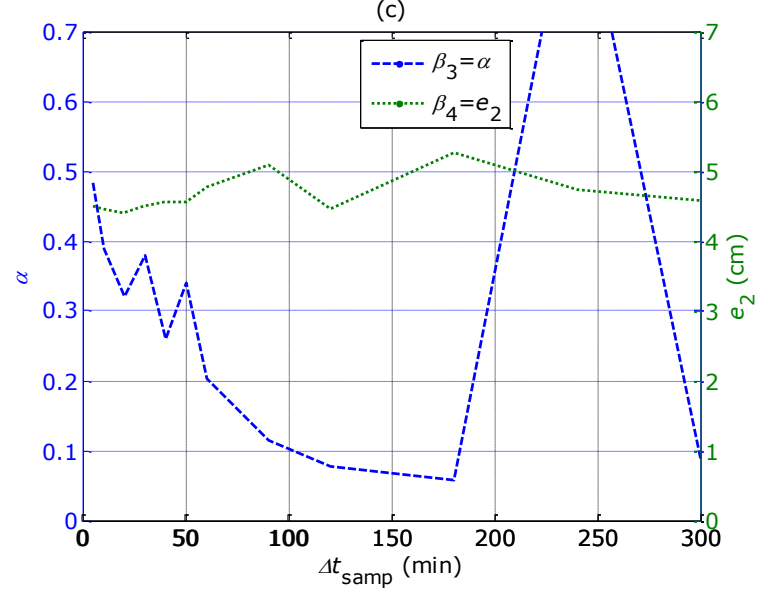

(b)

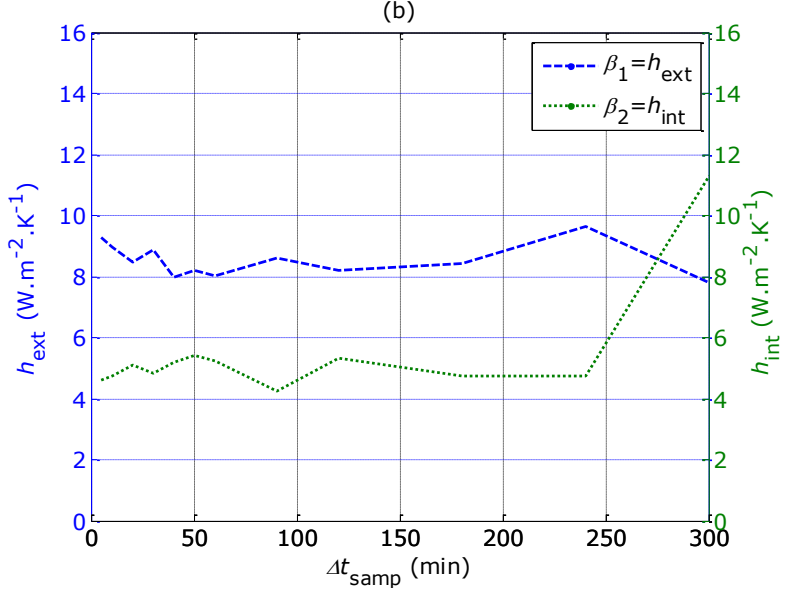

(d)

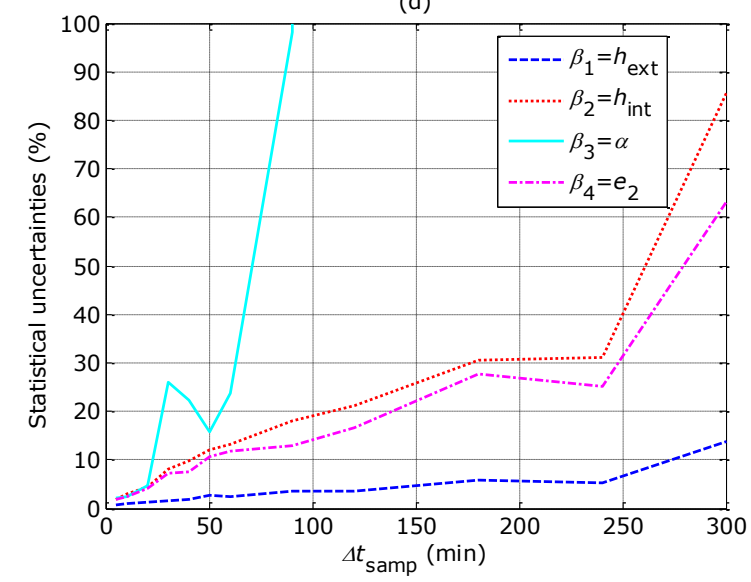

Figure 11. Identification results using the temperature gradient $\Delta T=T_{\text {int }}-T_{\text {ext }}$ as a function of sampling period: (a) residual norm, (b) estimated parameters $h_{\text {ext }}$ and $h_{\text {int, }}$ (c) estimated parameters $\alpha$ et $e_{2}$, (d) statistical uncertainties on estimated parameters

\section{Conclusion}

This study has shown that quantitative data providing information on the insulation level of a building wall could be obtained by using a survey of wall surface temperature using passive infrared thermography. Additional parameters such as indoor and outdoor air temperature and solar heat flux density are needed to complete infrared thermography measurements. Indeed, we showed the possibility to estimate either the thickness or the thermal conductivity of the insulating layer by analyzing the measurement data obtained for several consecutive days. Analysis duration and sampling period influences have also been investigated. It showed that a satisfactory estimation could be reached for an analysis duration of only one day or for a sampling period of several hours.

\section{Acknowledgements}

The authors want to thank the ADEME (French agency for environment and energy saving) for the financial support of the DPE-IITI project. The authors want also to thank the town of Noisiel (near Paris) for its participation to this project by giving us a free access to some public buildings to perform thermographic measurements.

\section{REFERENCES}

[1] M-H-A. Larbi Youcef, L. Ibos, V. Feuillet, Y. Candau, P. Balcon, A. Filloux, Quantitative diagnosis of insulated building walls of restored old constructions using active infrared thermography, QIRT Journal, vol. 8(1), 2011, p. 65-87.

[2] Albatici R., Tonelli A. M., "Infrared thermovision technique for the assessment of thermal transmittance value of opaque building elements on site", Energy and Buildings, vol. 42, 2010, p. 2177-2183.

[3] Ribarić S., Marčetić D., Vedrina D. S., "A knowledge-based system for the non-destructive diagnostics of façade isolation using the information fusion of visual and IR images", Expert Systems with Applications, vol. 36, 2009, p. 3812-3823.

[4] Marquardt D. W., "An algorithm for the least squares estimation of non linear parameters", SIAM Journal, vol. 1, 1963, p. 431441. 